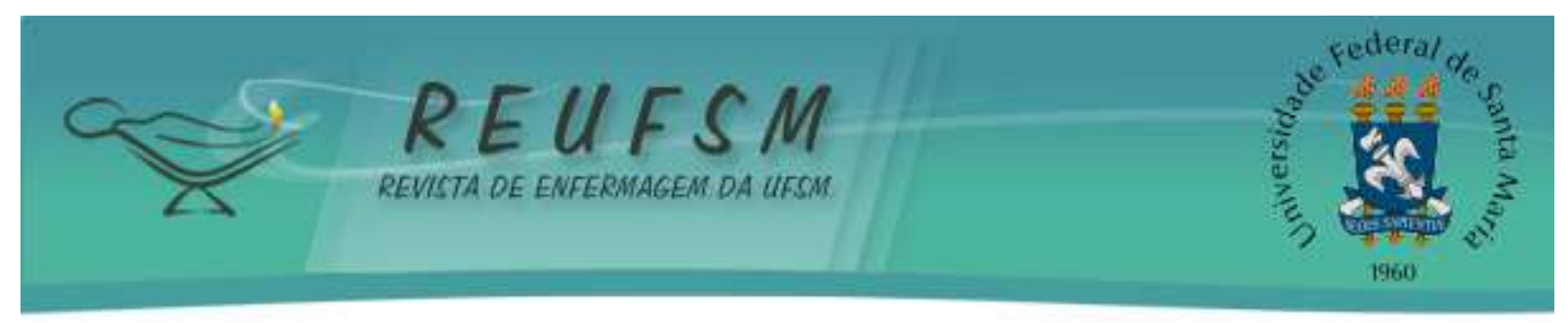

\title{
MANEJO DO HISTÓRICO DE ENFERMAGEM NO CONTEXTO DA TERAPIA INTENSIVA PEDIÁTRICA ${ }^{1}$
}

\section{NURSING HISTORY MANAGEMENT IN THE INTENSIVE PEDIATRIC THERAPY CONTEXT}

\section{ADMINISTRACIÓN DEL HISTÓRICO DE ENFERMERÍA EN EL CONTEXTO DEL CUIDADO INTENSIVO PEDIÁTRICO}

\author{
Jaynara Menezes Sousa Pinheiro ${ }^{2}$ \\ Francisca Georgina Macedo de Sousa ${ }^{3}$ \\ Andrea Cristina Oliveira Silva ${ }^{4}$ \\ Nicole Maria Costa e Silva ${ }^{5}$ \\ Danilo Marcelo Araújo dos Santos ${ }^{6}$
}

Doi: $10.5902 / 2179769228573$

RESUMO: Objetivo: caracterizar o manejo do Histórico de Enfermagem segundo o preenchimento pelos enfermeiros da Unidade de Terapia Intensiva pediátrica. Método: pesquisa documental retrospectiva utilizando 135 Históricos de Enfermagem de crianças internadas de janeiro de 2015 a janeiro de 2016. Utilizou-se instrumento construído a partir dos itens de cada Bloco do Histórico de Enfermagem do serviço contexto da pesquisa. Os escores variaram de 1 a 189 pontos e permitiram classificar o preenchimento como Péssimo (1 - 66 pontos); Ruim (67 - 100 pontos); Regular (101 - 145 pontos); Bom/Ótimo (145 ou mais pontos). Resultados: dos Históricos de Enfermagem analisados, 47,2\% foram classificados como preenchimento regular, $23,7 \%$ ruim, $22,2 \%$ ótimo e $6,9 \%$ péssimo. Conclusões: o manejo do Histórico de Enfermagem foi caracterizado como incipiente, ambivalente, ambíguo, incompleto e desqualificado no tocante à formalidade do seu preenchimento nas práticas de cuidado do enfermeiro intensivista.

Descritores: Enfermagem; Processos de enfermagem; Registros de enfermagem; Unidade de terapia intensiva pediátrica

ABSTRACT: Aim: to characterize the management of Nursing History according to the data filling made by the Pediatric Intensive Care Unit nurses. Methods: retrospective documentary research using 135 Nursing Histories of hospitalized children from January 2015 to January 2016. An instrument built from the items from each Nursing History Block of the research context

\footnotetext{
1 Manuscrito é parte da Dissertação de Mestrado defendida junto ao Programa de Pós-Graduação em Enfermagem da Universidade Federal do Maranhão (UFMA), vinculada ao projeto de pesquisa "Sistematização da Assistência de Enfermagem em Unidade de Terapia Intensiva Pediátrica: uma Pesquisa Convergente Assistencial", desenvolvido pelo Grupo de Estudo e Pesquisa na Saúde da Família, da Criança e Adolescente (GEPSFCA) com apoio financeiro do CNPq.

${ }^{2}$ Enfermeira. Mestre em Enfermagem. Enfermeira da Rede Sarah. São Luís, Maranhão, Brasil. E-mail: enf_jaynara@yahoo.com.br

3 Enfermeira. Doutora em Enfermagem. Docente do Curso de Enfermagem da Universidade Federal do Maranhão. São Luís, Maranhão, Brasil. E-mail: fgeorginasousa@ hotmail.com

${ }^{4}$ Enfermeira. Doutora em Ciências. Docente do Curso de Enfermagem da Universidade Federal do Maranhão. São Luís, Maranhão, Brasil. E-mail: andreacris09@hotmail.com

${ }^{5}$ Enfermeira. Graduada em Enfermagem pela Universidade Federal do Maranhão. São Luís, Maranhão, Brasil. E-mail: nicole_mcs@hotmail.com

${ }^{6}$ Enfermeiro. Mestre em Enfermagem. Enfermeiro do Hospital Universitário da Universidade Federal do Maranhão. São Luís, Maranhão, Brasil. E-mail: danilomasantos@ hotmail.com
} 


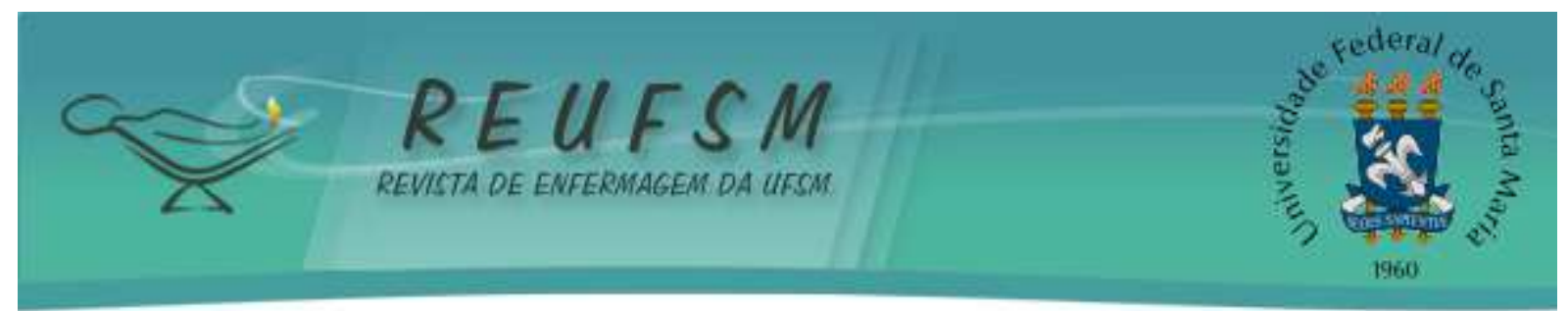

service was used. The scores ranged from 1 to 189 points and allowed the filling to be classified as Poor (1 - 66 points); Bad (67 - 100 points); Regular (101 - 145 points); Good / Great (145 or more points). Results: from the analyzed Nursing Histories, $47.2 \%$ were classified as regular, $23.7 \%$ bad, $22.2 \%$ great and $6.9 \%$ as poor. Conclusions: the management of Nursing History was characterized as incipient, ambivalent, ambiguous, incomplete and disqualified regarding the formality of its filling in the care practices of the intensive care unit nurse.

Descriptors: Nursing; Nursing process; Nursing records; Intensive care units, pediatric

RESUMEN: Objetivo: caracterizar la administración del Histórico de Enfermería según el relleno hecho por los enfermeros de la Unidad de Cuidado Intensivo pediátrico. Método: investigación documental retrospectiva basada en 135 históricos de Enfermería de niños internados entre enero de 2015 y enero del 2016. Para tanto, se utilizó un instrumento construido a partir de los ítems de cada Unidad del Histórico de Enfermería del servicio contexto de la investigación. Las calificaciones variaron de 1 a 189 puntos y permitieron clasificar el relleno como Pésimo (1 - 66 puntos); Malo (67 - 100 puntos); Regular (101 - 145 puntos); Bueno/Óptimo (145 o más puntos). Resultados: de los Históricos de Enfermería analizados 47,2\% fueron clasificados como relleno regular, 23,7\% malo, 22,2\% óptimo y 6,9\% pésimo. Conclusiones: la administración del Histórico de Enfermería fue caracterizado como incipiente, ambivalente, ambiguo, incompleto y descalificado con relación a la formalidad de su relleno en las prácticas de cuidado del enfermero intensivista.

Descriptores: Enfermeira; Proceso de enfermería; Registros de enfermería; Unidades de cuidado intensivo pediátrico

\section{INTRODUÇÃO}

A essência da enfermagem é o cuidar e a Sistematização da Assistência de Enfermagem (SAE) é a metodologia usada para planejar, executar e avaliar o cuidado. É caracterizada como instrumento imprescindível ao trabalho do enfermeiro por ampliar seu campo de atuação e evidenciar suas práticas por meio do conhecimento científico. ${ }^{1}$ Nesse âmbito, a enfermagem tem o Processo de Enfermagem (PE) como ferramenta de organização do trabalho, desencadeamento dos pensamentos e avaliação durante a prestação do cuidado. Operacionalmente, o PE é desenvolvido em etapas e envolve a identificação de problemas de saúde do cliente (Histórico), o delineamento do Diagnóstico de Enfermagem (DE), a instituição do Planejamento de Enfermagem (intervenção ou plano de cuidados), a implementação das ações prescritas e a Avaliação ou Evolução de Enfermagem. ${ }^{2-3}$

Nessa perspectiva, os cuidados de enfermagem são realizados de maneira integral e ampliada, e permitem ao enfermeiro sistematizar suas intervenções de forma clara e organizada, uma vez que rompe, em parte, com o modelo médico centrado, tecnicista, no qual 


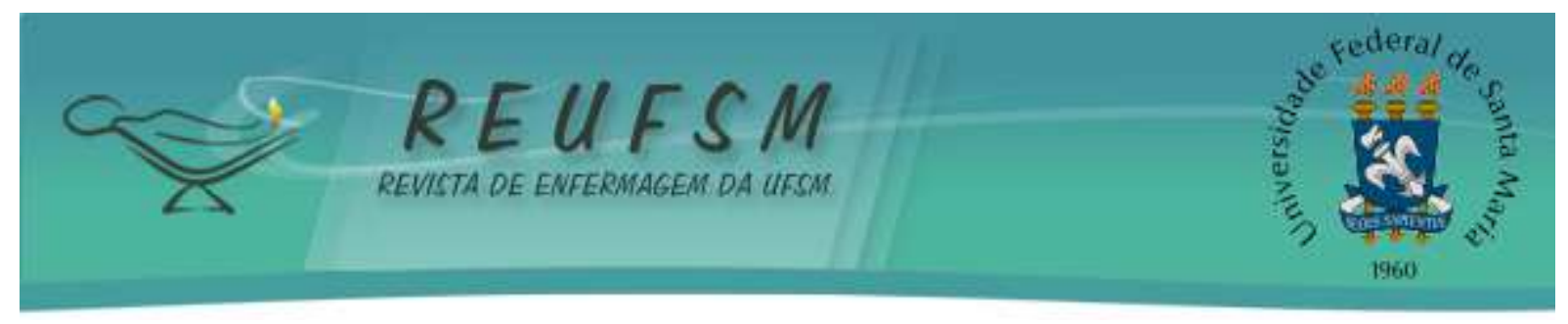

provê uma sequência lógica do raciocínio e guia para a adoção de um julgamento clínico e terapêutico adequado. ${ }^{4}$

Entende-se que para uma assistência holística e humanizada em Unidades de Tratamento Intensivo (UTI), onde há prática contínua de assistência especializada, torna-se necessário a implantação e execução da SAE, possibilitando ao enfermeiro intensivista organizar, planejar, intervir e solucionar alterações e complicações detectadas precocemente, visando à melhoria na qualidade de vida e à recuperação dos pacientes dessa unidade. ${ }^{5-6}$ Sob essa perspectiva e após construção e implantação do Histórico de Enfermagem na UTI Pediátrica de um Hospital Universitário ${ }^{7-8}$ da região nordeste do Brasil para atender a necessidade de continuidade na organização e documentação da Assistência de Enfermagem nesse serviço, emergiu como necessidade e urgência avaliar o manejo do Histórico de Enfermagem pelos enfermeiros que exercem suas atividades no contexto mencionado.

Sob esse escopo, a pesquisa foi guiada pela pergunta: qual a natureza qualitativa dos Históricos de Enfermagem da Unidade de Terapia Intensiva Pediátrica segundo o manejo realizado pelos enfermeiros desse serviço?

A relevância da investigação tem como base a afirmativa de que a implementação da SAE proporciona cuidados individualizados, assim como norteia o processo decisório do enfermeiro nas situações de gerenciamento da equipe de enfermagem, oportuniza avanços na qualidade da assistência e impulsiona sua adoção nas instituições que prestam assistência à saúde. Ressalta-se que a UTI Pediátrica contexto em tela, faz parte de um Hospital Universitário, portanto, a investigação trará benefícios não somente aos profissionais da área e pacientes, mas também ao corpo docente e discente que atuam nesse local.

Foi elencado como objetivo caracterizar o manejo do Histórico de Enfermagem segundo o preenchimento pelos enfermeiros da UTI Pediátrica.

\section{MÉTODO}

Trata-se de parte de investigação intitulada "Sistematização da Assistência de Enfermagem em Unidade de Terapia Intensiva Pediátrica: uma pesquisa convergente assistencial", desenvolvida em três etapas que compreendeu a construção, a implantação e a avaliação do manejo do Histórico de Enfermagem (HE). As duas primeiras etapas foram 


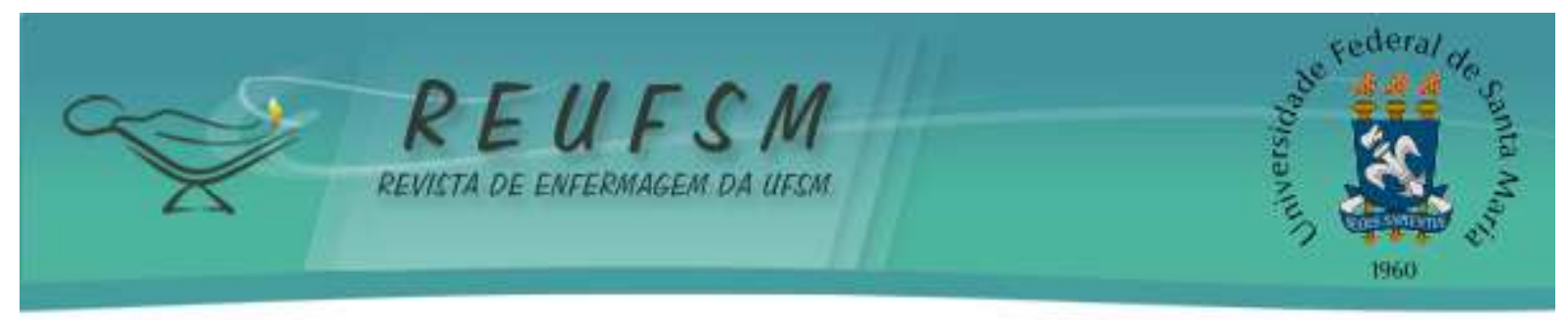

realizadas no período de 2013 a 2014 e, após 12 meses de implantação do HE foi realizada a terceira etapa que compreendeu o manejo do referido Histórico, objeto deste manuscrito.

Para avaliar o manejo do HE construído e implantado em etapa anterior, utilizou-se de estudo retrospectivo, transversal e documental com abordagem quantitativa. A coleta de dados foi realizada com dados secundários tendo como base os HE da UTI Pediátrica, no período de janeiro de 2015 a janeiro de 2016.

Enfatiza-se que a Teoria das Necessidades Humanas Básicas de Wanda de Aguiar Horta foi selecionada para apoiar a construção do HE implantado na UTI Pediátrica, em virtude de permitir a avaliação do paciente como um todo indivisível, reforça a importância do cuidado ao ser humano e sua estrutura é centrada nas necessidades básicas afetadas. E, ainda, pelo fato de ser esta a teoria escolhida e adotada para embasar a SAE em todos os setores do Hospital Universitário, foco desta investigação.

Para localização dos HE foi necessário identificar as internações da UTI Pediátrica no período definido. Para tanto, foram utilizados os registros de admissão e o sistema informatizado no qual consta número de prontuário e data da internação. Desse processo foram identificados 248 prontuários, entretanto, 11 deles não foram acessados (02 por óbito e 09 estavam no trâmite legal de faturamento); três (03) foram excluídos, pois serviram como teste piloto para utilização do instrumento de coleta de dados, totalizando 234 prontuários. Desse total 99 estavam sem o HE anexado à documentação, portanto, 135 prontuários serviram de base para a análise dos dados.

Os HE localizados foram analisados com apoio de um instrumento construído especificamente para esse fim, composto por 106 itens seguindo a mesma estrutura do HE implantado na UTI Pediátrica (desenvolvido e estruturado em sete blocos: Identificação; Antecedentes; História da Doença Atual; Necessidades Psicobiológicas; Necessidades Psicossociais; Necessidades Psicoespirituais; e Outros dados relevantes e observações do Enfermeiro em etapa anterior da pesquisa). ${ }^{8}$

$\mathrm{O}$ instrumento é do tipo checklist, disposto em um quadro com cinco colunas. $\mathrm{Na}$ primeira constava a descrição do item e nas colunas seguintes as possibilidades de resposta segundo a avaliação do preenchimento do referido item: PC (preenchimento completo), PI (preenchimento incompleto), NP (não preenchido) e NA (não se aplica), as quais deveriam ser assinaladas com " $\mathrm{X}$ " de acordo com o que constava no HE. A análise do preenchimento do 


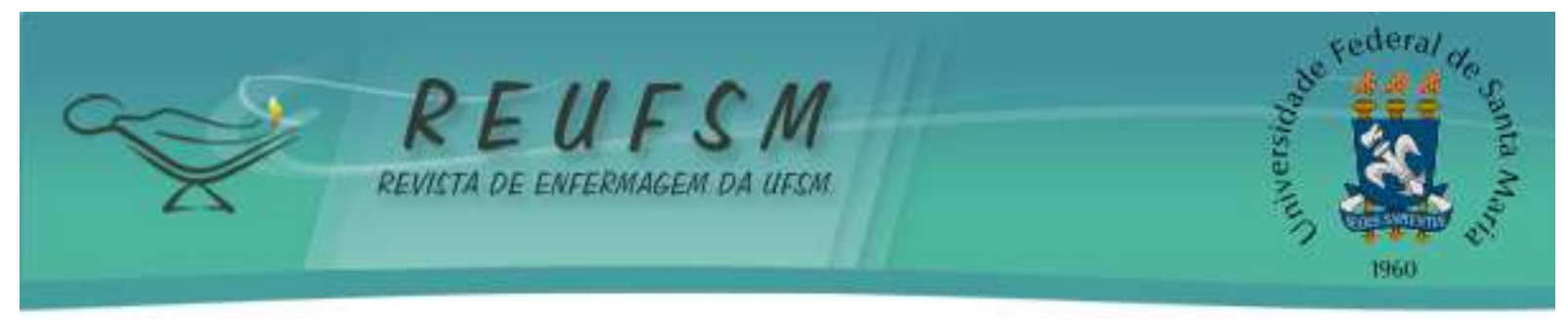

HE deu-se a partir de duas possibilidades: pela descrição dos itens com preenchimento adequado e a partir de um escore para avaliação similar ao usado em estudo anterior que classificou o HE em péssimo, ruim, regular, bom ou ótimo, que será descrito a seguir. ${ }^{9}$

Assim, foi atribuído peso de 1 a 3 segundo os itens do HE: Peso 1 para os itens da Identificação, Antecedentes e Histórico da Doença Atual; Peso 2 para os itens referentes às Necessidades Psicobiológicas, Psicossociais e Psicoespiritual; Peso 3 para os itens referentes ao Enfermeiro responsável pelo preenchimento do HE. Em seguida, foram classificados como "péssimo" (1 a 65 pontos - 1\% a 35\%); "ruim" (66 a 99 pontos - 36\% a 53\%); "regular" (100 a 144 pontos $-54 \%$ e $77 \%$ ); "bom ou ótimo" a partir de $78 \%$ (145 ou mais pontos). ${ }^{9}$

A pesquisa assegurou os preceitos éticos de acordo com as orientações e disposições contidas na Resolução 466/2012 do Conselho Nacional de Saúde ${ }^{10}$ e foi aprovada sob protocolo de número 258.249 pelo Comitê de Ética em Pesquisa do Hospital Universitário da Universidade Federal do Maranhão, no dia 19 de abril de 2013.

\section{RESULTADOS}

Os resultados serão apresentados em tabelas em números relativos e percentuais e as inconsistências dos registros são descritos segundo cada item e sessão do Histórico de Enfermagem.

Tabela 1 - Distribuição dos prontuários da UTI Pediátrica localizados de acordo com a presença ou ausência do Histórico de Enfermagem

\begin{tabular}{crc}
\hline Prontuários & n & \% \\
\hline Com Histórico de Enfermagem & 135 & $57,7 \%$ \\
Sem Histórico de Enfermagem & 99 & $42,3 \%$ \\
\hline TOTAL & 234 & $100 \%$ \\
\hline
\end{tabular}

Apesar de 57,7\% dos prontuários apresentarem o $\mathrm{HE}$, chama atenção o número de prontuários sem que os enfermeiros tenham realizado a primeira etapa de Processo de Enfermagem (42,3\%). Ressalta-se que, a pesquisa para avaliação e caracterização do manejo do HE por enfermeiros da UTI Pediátrica foi realizada 1 ano após implantação dessa etapa na unidade. Esse dado pode revelar o pouco envolvimento dos profissionais enfermeiros com o HE, ou ainda que as questões que envolveram aposentadoria, demissão e inserção de novos profissionais no quadro da UTI nesse período, tenham sido determinantes para estes resultados. 


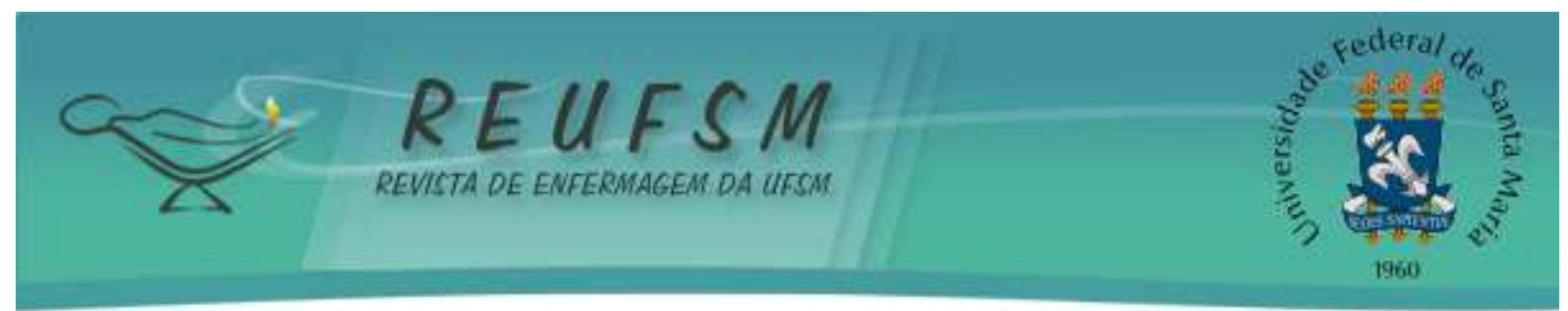

Tabela 2 - Classificação do Histórico de Enfermagem da UTI Pediátrica segundo escore de avaliação do preenchimento dos itens de cada Bloco

\begin{tabular}{ccc}
\hline $\begin{array}{c}\text { Classificação do Preenchimento } \\
\text { do HE }\end{array}$ & $\mathbf{n}$ & $\%$ \\
\hline Péssimo & 05 & $3,7 \%$ \\
Ruim & 22 & $16,3 \%$ \\
Regular & 73 & $54,0 \%$ \\
Bom ou Ótimo & 35 & $26,0 \%$ \\
\hline TOTAL & 135 & $100 \%$ \\
\hline
\end{tabular}

O preenchimento regular do HE foi o de maior percentual $(54,0 \%)$ seguido por aqueles caracterizados como bom ou ótimo (26,0\%). O quantitativo dos HE classificados como ruim $(16,3 \%)$ e péssimo $(3,7 \%)$ totalizam $20 \%$ com registros inconsistentes e de forma incompleta.

\section{Inconsistências nos Registros do Histórico de Enfermagem}

a) Bloco I - Identificação

Neste bloco, quase a totalidade dos itens apresentaram preenchimento completo, com destaque para o nome da criança 98,5\%; data de nascimento 93,3\%; sexo 95,5\%; idade 83,7\%; admissão no hospital 86\%; admissão na UTI Pediátrica 83\%; nome da mãe 87,4\%; parentesco com a criança 80,8\%; endereço77\% e identificação do leito 72,6\%. Itens não preenchidos: Grupo $\mathrm{ABO}$; número de telefone para contato com a família; escolaridade; cor/raça; frequência em creche ou escola. A ausência dessas informações pode comprometer o plano de cuidados e a comunicação com a família.

b) Bloco II - Antecedentes (doenças de base, doenças de infância, internações anteriores, medicações em uso, antecedentes familiares e hábitos de vida):

$\checkmark$ Doença de base: 71,1\% dos HE foram preenchidos completamente, 5,9\% com dados incompletos e $23 \%$ não preenchidos;

Doenças da infância: $74 \%$ preencheram o campo completamente, porém com muitos equívocos, pois consideraram anemia, cardiopatia, convulsão, gripe, nefropatia e pneumonia como doenças da infância. No registro das internações anteriores, 78,5\% dos HE estavam com registro completo, 9,6\% incompletos, faltando o motivo da internação;

$\checkmark$ Medicações em uso: 76,3\% apresentaram registro completo. Em 5,2\% o enfermeiro assinalou que a criança fazia uso de medicações, mas sem especificação;

Antecedentes familiares: 73,3\% dos HE apresentaram registro completo e em 21,5\% não houve registro deste item; 


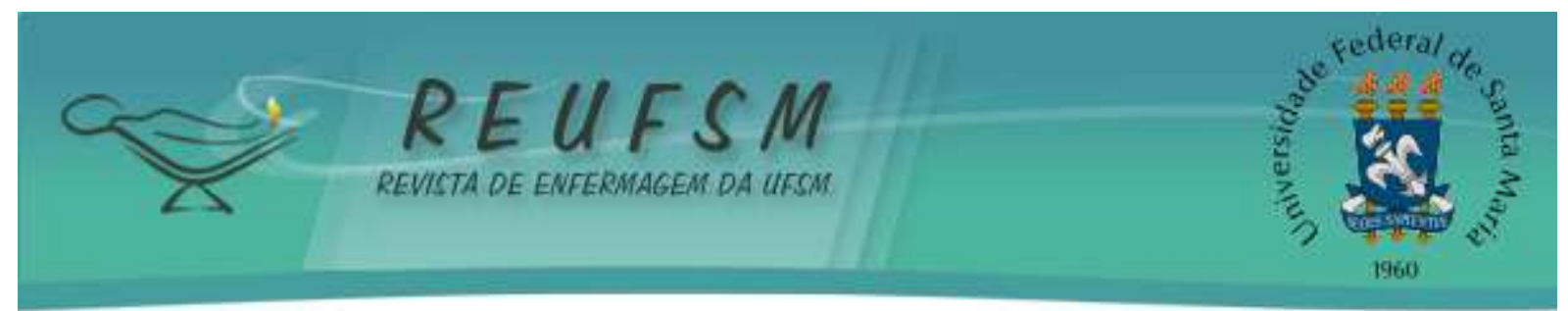

$\checkmark$ Hábitos de Vida da criança: sem registro em 48,2\% dos HE e 51,1\% deles foi preenchido completamente, porém com algumas inconsistências, entre elas, considerar "estudante" e "doença da criança" como hábitos de vida.

c) Bloco III - História da Doença Atual (queixa principal, início dos sinais/sintomas, evolução da doença e tratamento atual):

$\checkmark$ Queixa principal: dos 135 HE analisados $85,9 \%$ foram caracterizados com preenchimento completo e os incompletos e não preenchidos totalizaram 14,1\%;

$\checkmark$ Início dos sinais/sintomas: ausente desse registro em 27,4\% dos HE;

$\checkmark$ Evolução da doença: a informação foi preenchida completamente em $65,2 \%$ dos HE, sendo que em $11,8 \%$ o espaço estava em branco;

$\checkmark$ Tratamento atual: refere-se ao detalhamento da conduta terapêutica utilizada no momento da internação. Em 71,1\% dos HE a informação estava completa, mas com inconsistências como abreviaturas e sem especificação do tratamento.

d) Bloco IV - Necessidades Psicobiológicas

$\checkmark \mathrm{Na}$ seção oxigenação, os itens com preenchimento completo foram: padrão respiratório (93,3\%); frequência respiratória $(86,7 \%)$; e ventilação mecânica $(83,7 \%)$. Apesar de alguns enfermeiros marcarem a modalidade ventilação mecânica, não a caracterizaram;

$\checkmark$ Ausculta pulmonar: $64,4 \%$ preenchimento incompleto pela ausência de informações como murmúrios vesiculares, ruídos adventícios e, quando colocaram ruídos adventícios, não os localizaram;

$\checkmark$ Tosse: 89,6\% esse item foi preenchido. Por ser um item cujo registro pode ser caracterizado como simples, pois consiste em assinalar SIM ou NÃO, e, em seguida, caracterizar se produtiva ou improdutiva e a característica da secreção, não totalizou 100\%;

$\checkmark$ Frêmito toracovocal e percussão foram itens não preenchidos;

$\checkmark \mathrm{Na}$ seção Hidratação/Nutrição/Eliminação/Regulação Hidroeletrolítica: registros incompletos e ausentes;

Eliminações intestinais: em $29,7 \%$ dos $\mathrm{HE}$ esse registro não foi realizado. Nesse campo, deveria ser preenchida a frequência, o aspecto e outras características das eliminações intestinais, incluindo a data da última evacuação;

Na diurese: $60 \%$ de registros incompletos devido a não especificação dos dispositivos e caracterização da sonda vesical de demora; 


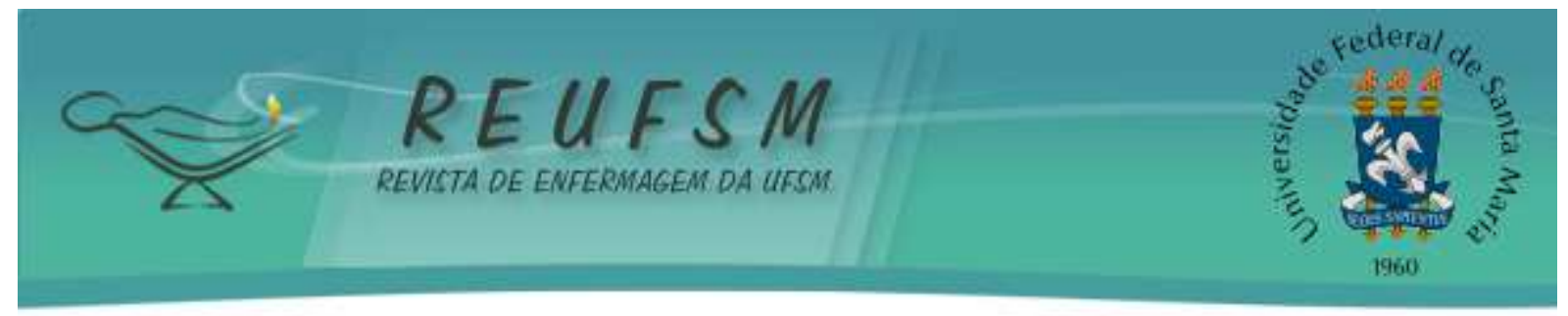

$\checkmark$ Regulação Neurológica/Exercício e Atividade Física/Mobilidade/Sono e Repouso/Mecânica Corporal foi o item com maior percentual de registro completo. Porém, alguns itens, como Escala de Glasgow, o registro não foi realizado. Ainda nessa seção, o nível de consciência, apesar de quase a totalidade $(98,5 \%)$ dos registros apresentarem-se completos, havia inconsistências como marcação simultânea de sedado e inconsciente e de sedado e orientado. A maior frequência de registro não preenchido correspondeu ao campo que descrevia mobilidade $(59,3 \%)$;

$\checkmark$ Quanto as condições cardiovasculares, os enfermeiros deveriam registrar inicialmente, a frequência cardíaca (FC), a pressão arterial (PA), a Pressão Arterial Invasiva (PAI), Pressão Arterial Pulmonar (PAP) e a Pressão Venosa Central (PVC), essas três últimas se a criança/adolescente estivesse sendo monitorada, caso contrário, passariam um traço no local destinado ao registro da informação. Os dois primeiros itens (FC e PA) tiveram seus valores registrados em 95,6\% e 81,5\%, respectivamente. Apesar dessa informação ser adquirida diretamente dos monitores em 4,4\% dos HE não constava o registro da FC e $17 \%$ da PA; no registro do pulso, $34,8 \%$ dos $\mathrm{HE}$ os enfermeiros marcaram apenas a intensidade ou regularidade, o que tornou o registro incompleto. No espaço referente a bulhas $34,1 \%$ dos $\mathrm{HE}$ estavam com registro completo, ficando a maior parte deles, $65,2 \%$ sem esse registro. $\mathrm{O}$ último item analisado da seção cardiovascular diz respeito a presença de dispositivos e drenos cujo percentual de registros completos foi de $60,7 \%$;

$\mathrm{Na}$ seção Cuidado corporal/Integridade física/cutaneomucosa e Segurança física, o item referente à higiene bucal e integridade do couro cabeludo foi o de maior registro de preenchimento completo, ambas com 94,8\%; a pele apresentou 51,9\% de registro incompleto, ocorrido pelo não registro da coloração ou da integridade da pele;

$\checkmark$ Quanto aos itens Abrigo, Ambiente e Regulação Térmica o enfermeiro deveria assinalar o tipo de acomodação da criança se berço aquecido ou cama ou se estava ou não em isolamento. $\mathrm{O}$ resultado foi que $77 \%$ dos $\mathrm{HE}$ foram caracterizados com preenchimento completo, $16,3 \%$ como incompleto e 6,7\% não preenchido. Dos incompletos, não constava especificação se a criança estava ou não em isolamento; na regulação térmica, 79,3\% dos HE se apresentou completo, $12,6 \%$ incompleto e $8,1 \%$ não preenchido;

$\checkmark$ Em relação a seção Regulação hormonal, Crescimento celular e Sexualidade grande número dos $\mathrm{HE}$ estava sem preenchimento neste item $(79,3 \%)$; as medidas dos perímetros 


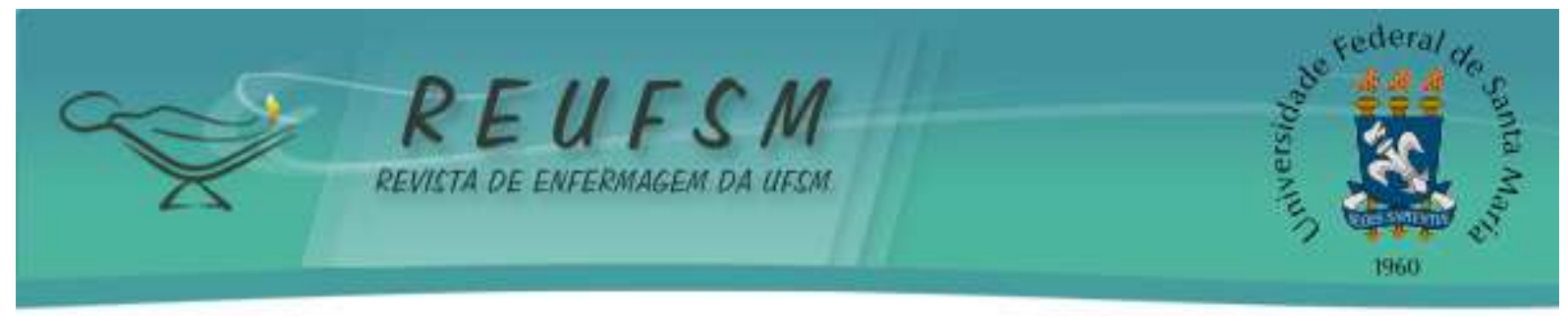

cefálico, torácico e abdominal foram preenchidas em 43,7\% dos HE. Em $83 \%$ o registro do peso foi completo e em $17 \%$ essa informação não foi registrada. Por consequência, a ausência dessa informação gerou a não informação no campo do Índice de Massa Corpórea e Escore-Z, importantes para a classificação dos indicadores de crescimento;

Na seção Regulação Imunológica 64,4\% dos registros foram caracterizados como completos, $26,7 \%$ incompletos e 8,9\% não preenchidos;

$\checkmark$ Na avaliação da dor os enfermeiros deveriam assinalar se a criança localizava estímulo doloroso por relato verbal ou pela expressão facial. Nesse item 54,8\% dos HE apresentaram registro completo, $40 \%$ incompletos e 5,2\% não preenchido.

e) Bloco V - Necessidades Psicossociais:

Este bloco é subdividido em cinco seções: Amor/Gregária; Comunicação, Atenção, Educação à Saúde; Aceitação, Autoestima, Autorealização, Autoimagem, Segurança Emocional; Recreação, Lazer, Sociabilidade, Participação, Criatividade, Liberdade e Espaço.

Referente a seção Amor/Gregária, 24,4\% dos registros foi caracterizado como completo, $68,1 \%$ incompleto e 7,4\% essa informação não foi preenchida;

$\checkmark$ Na Comunicação, Atenção e Educação à Saúde, a soma dos registros incompletos e não preenchidos foi de $48,2 \%$;

Na Aceitação, Autoestima, Autorrealização, Autoimagem e Segurança Emocional os registros no HE estavam completos em $77,8 \%$ deles;

Quanto aos itens Recreação, Lazer, Sociabilidade, Participação, Criatividade e Liberdade, $44,4 \%$ estavam completos e $21,5 \%$ desse campo não preenchido;

$\checkmark$ No item Espaço estão as opções residência, número de moradores ou cômodos, tipo de moradia, área, água tratada, eletricidade, coleta de lixo e rede de esgoto, 51,9\% apresentaram registros completos, $36,3 \%$ incompletos e $11,8 \%$ não preenchidos.

f) Bloco VI - Necessidades Psicoespirituais:

Este Bloco é constituído pelos itens: Religião; Frequência que participa das atividades religiosas; necessita da presença de líder espiritual e Desejo de ser batizado. Nas respostas deveriam constar: o tipo de religião praticada pelo paciente ou família; relatar a assiduidade em atividades religiosas; se necessitava ou não de líder espiritual; se era batizado, se não fosse, se desejaria ser. Do total 56,3\% estavam completos e 33,3\% incompletos.

g) Bloco VII - Dados Relevantes e Observações do Enfermeiro: 


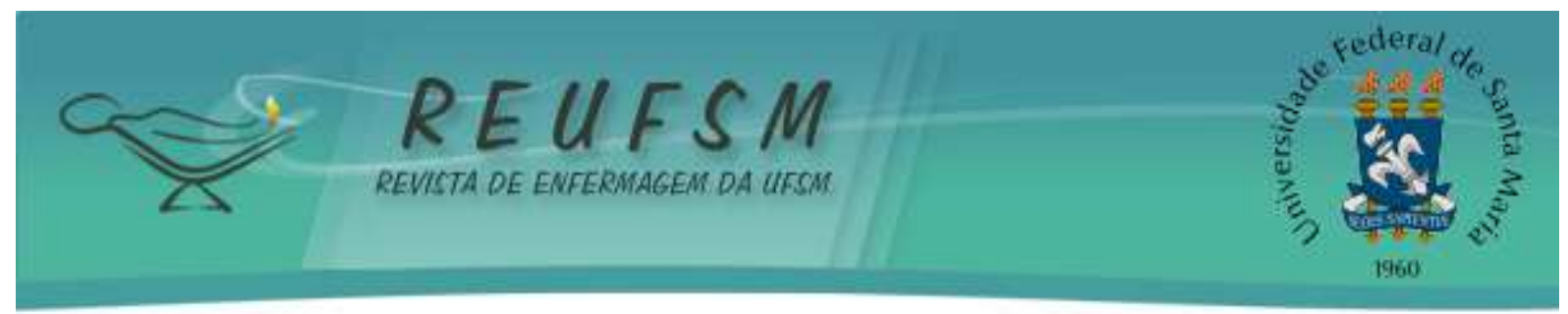

Este espaço destina-se ao registro de informações identificadas e consideradas importantes pelo enfermeiro e que não foram contempladas nos blocos que estruturam o Histórico de Enfermagem. Em seguida, o enfermeiro deveria datar o HE utilizando o padrão dia/mês/ano, referir a hora do preenchimento, assinar e carimbar:

No tocante ao espaço destinado às observações e dados relevantes $43,7 \%$ dos $\mathrm{HE}$ estavam completamente preenchidos, entretanto 56,3\% dos HE não havia nenhum registro do Enfermeiro responsável pelo preenchimento do referido instrumento;

$\checkmark$ Quanto à identificação do enfermeiro (registro do nome, do número do Conselho Regional de Enfermagem (COREN), carimbo, data e hora de preenchimento), constava o registro completo desses itens, 90,4\%, 67,0\%, 66,7\% e 78,5\%, respectivamente. Até mesmo nesse item foram encontrados HE sem assinatura e sem carimbo do enfermeiro.

\section{DISCUSSÃO}

No processo de cuidar da saúde, a documentação e o registro escrito das atividades desenvolvidas é instrumento de extrema valia para o planejamento, execução, continuidade e avaliação do serviço prestado, visando, sobretudo, a identificação dos problemas reais ou potenciais do paciente e para atender as necessidades identificadas. Nesta pesquisa pode-se inferir que a documentação e os registros de enfermagem não atendem a estas exigências e, que essa condição poderá comprometer a continuidade da assistência de enfermagem.

Foi frequente o número de registros incompletos ou a ausência destes referente à identificação da criança. No Bloco I (Identificação) foi identificado um percentual elevado de nomes das crianças/adolescentes internados na UTI redigidos com abreviaturas e sem o número do prontuário. Este fato é inaceitável quando se trata de registros oficiais, pois poderão comprometer o processo de comunicação e concorrer para risco da segurança do paciente.

Nesse bloco foi encontrado também o uso equivocado do termo "moreno" como cor/raça além de um dado preocupante quanto à baixa adesão ao registro dos sistemas $\mathrm{ABO}$ e $\mathrm{Rh}$, pelo fato de serem importantes recursos para a terapêutica na UTI em função de intercorrências clínicas que necessitam de suporte de transfusões sanguíneas ou para seleção de doadores. ${ }^{11}$ Assim, os registros devem conter o máximo de informações corretas e completas sobre o estado de saúde dos pacientes, sejam referentes aos procedimentos realizados, suas necessidades e suas queixas. ${ }^{12}$ 


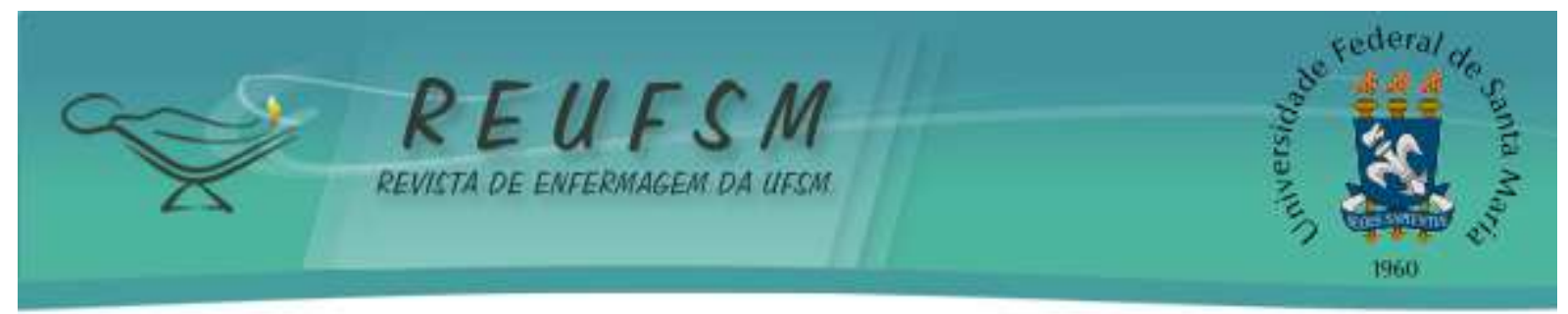

Ressalta-se que o registro escrito é uma das provas mais valiosas sobre a atuação e a qualidade dos cuidados prestados e que, quando são escassos e inadequados, comprometem a assistência prestada ao paciente e desqualificam a instituição e a equipe de enfermagem. A inexistência de registros ou a presença de lacunas nesse processo pode indicar uma má qualidade da assistência de enfermagem ${ }^{13}$ e revelar pouco compromisso dos enfermeiros com o instrumento de coleta de dados e com o Processo de Enfermagem.

Entretanto, para permitir a continuidade do planejamento dos cuidados de enfermagem, segundo a SAE, em suas diferentes fases, o registro deve ser devidamente identificado com dados completos do paciente, data e horário específicos, de forma legível e sem rasuras. Essa deve ser a preocupação contínua de enfermeiros e técnicos de enfermagem ${ }^{14}$ em qualquer contexto da atenção em saúde, pois além de fornecer elementos para o planejamento do cuidado, possibilita auditoria dos processos de enfermagem.

As inconsistências relativas ao não preenchimento dos dados ou a sua incompletude se repetiu nos itens que compõem o Bloco II (referente aos Antecedentes) e o Bloco III (História da Doença Atual).

Destaca-se o item padrão respiratório pela sua importância para o equilíbrio hemodinâmico da criança/adolescente, assim como para as ações de cuidado em terapia intensiva. Nesse item o enfermeiro deveria preencher com $X$ o padrão respiratório (superficial, profundo, eupneia, dispneia, bradipneia, taquipneia, batimento de asa de nariz, apneia, tiragem intercostal e músculos acessórios). Entretanto, os registros foram inconsistentes e incoerentes, como por exemplo: no item ventilação mecânica e dispositivos os enfermeiros assinalaram que a criança estava em ventilação espontânea, mas referiam o valor da FiO2, invalidando, mais uma vez, a informação. Na ausculta pulmonar, 20,8\% estavam com registro incompleto. Desses, o campo murmúrios vesiculares, ruídos adventícios ou ausculta não foram contemplados.

Entretanto, qualquer que seja o perfil dos pacientes, o enfermeiro deve estar atento ao atendimento das possíveis intercorrências que podem ocasionar mudanças na homeostase orgânica, dentre as quais as arritmias cardíacas e o desconforto respiratório ${ }^{15}$ definindo-se como informações relevantes para a avaliação do paciente em terapia intensiva.

No tocante a diurese, o maior quantitativo de HE estava com registros incompletos $(62,5 \%)$, devido a não especificação dos dispositivos e caracterização da sonda vesical de 


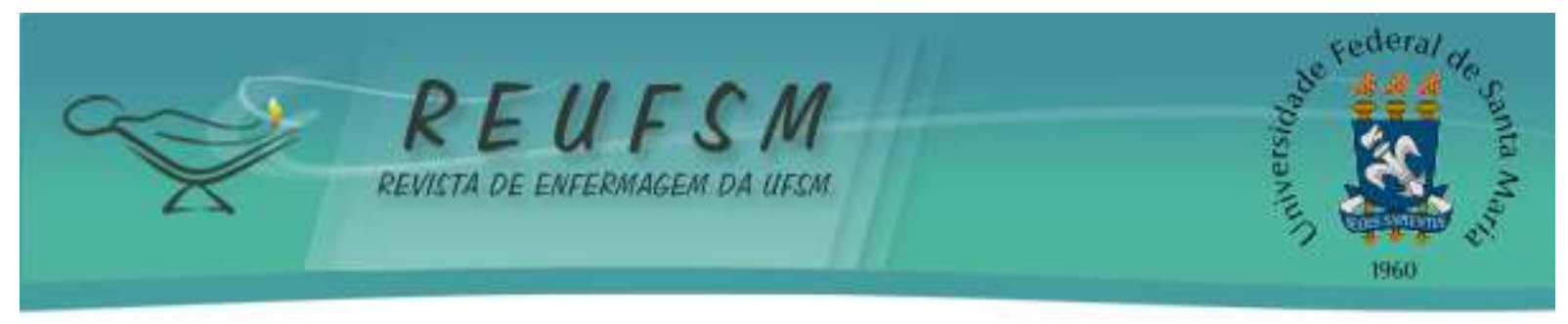

demora. Torna-se indispensável o registro do item diurese, pois a maioria dos casos de infecção do trato urinário (ITU) hospitalar ocorre após procedimento de cateterização do trato urinário, por exemplo. No espaço referente a bulhas, teriam que descrever as características das bulhas cardíacas. Somente 19,4\% dos HE estavam com registro completo, ficando 79,2\% sem esse registro, sugerindo que o enfermeiro não realizou ausculta cardíaca quando da admissão da criança na UTI.

Acerca do acesso venoso, destaca-se que 47,2\% dos HE foram definidos como registros incompletos, pois só foi assinalada a opção do acesso, mas não caracterizava ou descrevia a localização. Essa informação facilita a detecção de extravasamento e infiltração no acesso venoso, ficando a equipe de enfermagem responsável por prevenir e detectar, precocemente, os fatores de risco para estas complicações, oferecendo os cuidados para sua prevenção e tratamento.

Defende-se que o planejamento dos cuidados de enfermagem fundamentado nas especificidades de cada indivíduo, é indispensável no contexto da terapia intensiva, pois características peculiares e instabilidades hemodinâmicas, na maioria dos casos, são identificadas pela realização do exame físico.

Relacionado ao cuidado corporal, a ausência de registros preocupa porque mesmo sendo cuidados considerados de rotina, têm importância para o bem-estar e recuperação do paciente. ${ }^{16}$ Além disso, a falta de informação indica que os cuidados de higiene não são valorizados pela equipe.

Diante da incompletude ou ausência dos dados referentes ao Bloco V (Necessidades Psicoespirituais), asseverou-se que os enfermeiros demonstraram pouca preocupação em relação à coleta e análise de dados voltados para as necessidades psicossociais, fato que pode estar relacionado às dificuldades de avaliação das condições subjetivas do paciente durante a execução do Histórico de Enfermagem.

No Bloco VII estão os Dados Relevantes e Observações do Enfermeiro. Nele as lacunas relativas ao preenchimento na identificação do Enfermeiro foram também citadas em outra pesquisa, cujos registros de enfermeiros e auxiliares de enfermagem, em sua maioria, não foram identificados com o nome do profissional, data e hora do registro. ${ }^{17}$ Os registros não são apenas importantes para a segurança do paciente, mas para o respaldo legal do profissional que realizou a assistência adequada e de qualidade. ${ }^{18}$ 


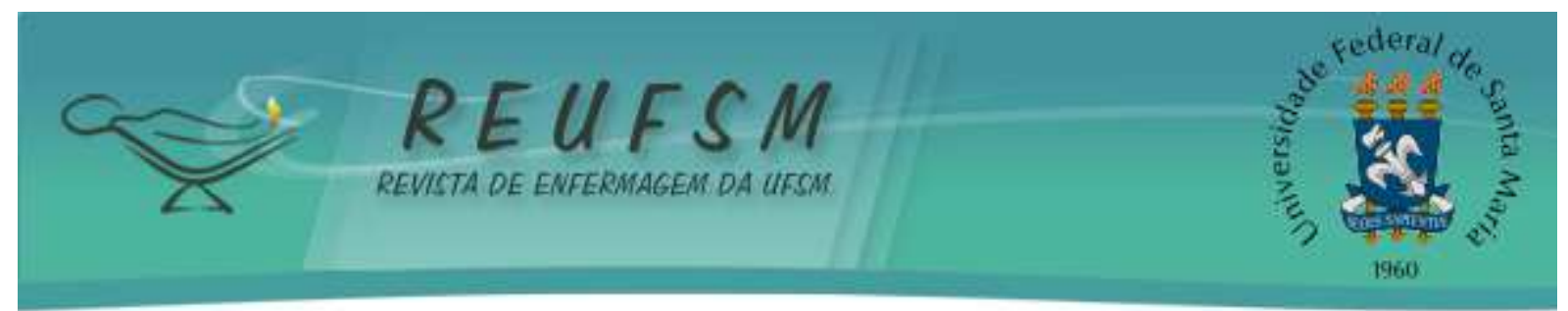

Constatou-se que no manejo do HE da UTIP pesquisada nenhum dos blocos apresentava registro completo, identificando-se registros falhos, incompletos ou em branco. Por outro lado, a coleta de informações realizada por meio do HE é a etapa que irá fundamentar o julgamento crítico do enfermeiro no reconhecimento de evidências apresentadas pelo paciente. Essas informações serão confrontadas com normas e critérios reconhecidos e recuperados da memória, sendo realizadas inferências que subsidiarão a construção dos diagnósticos de enfermagem. ${ }^{19}$ Portanto, informação não registrada é informação perdida e a escassa documentação sugere má prática na enfermagem. Ao contrário, os registros de enfermagem devem ser completos, redação qualificada e sem rasuras pois, acima de tudo, indicam qualidade do cuidado de enfermagem.

Entretanto, o paradigma que preside o modelo de atenção na UTI é fortemente determinado pela medicalização e pelo recurso tecnológico que, apesar de importante e indispensável, não atende isoladamente os desafios da Enfermagem científica no qual enfermeiros e técnicos de enfermagem estão imersos, cotidianamente, em procedimentos sobrando-lhes pouco tempo ou quase nada, para pensar e construir novas práticas. Estas assertivas são opositores para a SAE mas, ao enfrentá-las, enfermeiros e técnicos de enfermagem poderão ampliar e qualificar as ações de cuidado.

\section{CONCLUSÃO}

A pesquisa permitiu identificar como o manejo do Histórico de Enfermagem tem sido conduzido pelos enfermeiros da UTI Pediátrica por meio da análise da qualidade dos registros. Estes foram definidos como incipientes quanto às informações necessárias ao levantamento de dados da criança gravemente enferma. Essa condição acarreta, na maioria das vezes, atraso na tomada de decisões, considerando que há muitas lacunas nas informações dos pacientes.

Nenhuma dimensão do Histórico de Enfermagem foi preenchida completamente, sequer o bloco de Identificação. Com isso, torna-se fundamental, que os enfermeiros redimensionem sua prática assistencial e a maneira de registrar as informações, pois o Histórico de Enfermagem apoiado na Teoria das Necessidades Humanas Básicas de Wanda de Aguiar Horta permite ao enfermeiro planejar o cuidado individualizado e ampliado ultrapassando a dimensão biológica e o automatismo tão presentes no contexto da terapia intensiva. 


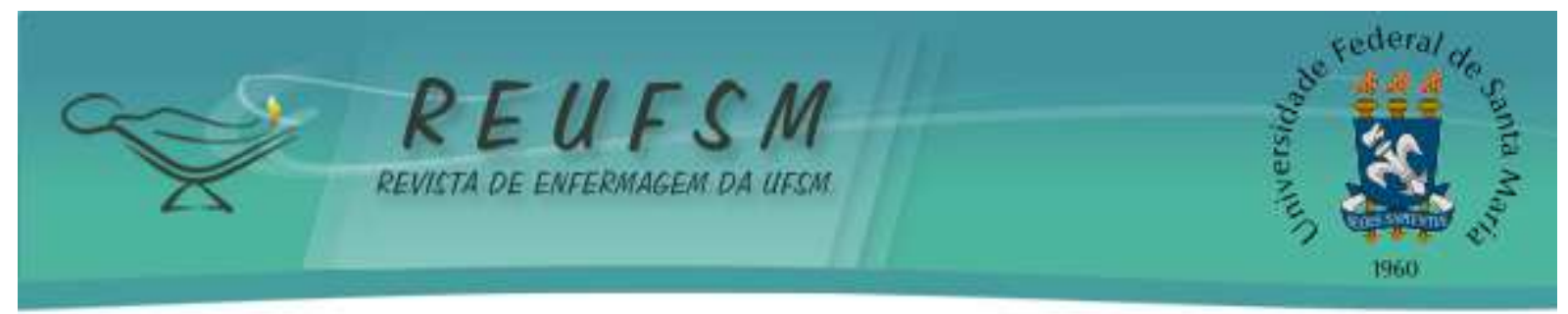

Espera-se que estes resultados possam contribuir tanto para o serviço como para a vida profissional dos enfermeiros e que estes tenham propósitos mais consistentes e valorativos da prática da enfermagem e suscite reflexões sobre a forma da assistência e o registro da mesma, valorizando a SAE e suas etapas, em especial, o Histórico de Enfermagem.

Será ainda necessário considerar que o mundo do trabalho é lugar de reflexão, de espaço de diálogos, de cooperação e de produção de novos acordos possíveis de tornar a SAE objeto prioritário para ampliar a responsabilização e a continuidade do cuidado integral. Por isso, o apoio à organização e a mobilização de atores é vital, pois a SAE é produção do espaço micropolítico das práticas de enfermagem. É possível compreender novas leituras observando os contornos do trabalho de enfermagem em UTI Pediátrica por meio da SAE, favorecendo aos trabalhadores dessa importante área da saúde, uma ação crítica, reflexiva e criativa. É factível admitir que os enfermeiros imersos na engrenagem cotidiana do modelo biomédico constroem limitadores para sua própria prática que o impele, incessantemente, à reprodução de técnicas e que legitima imposições e comportamentos repressores.

Há uma urgência em tornar a SAE uma prática central nos contextos hospitalares, sejam eles públicos ou privados. Mas, essa ação deverá estar intimamente vinculada à produção do cuidado, ao compromisso e à autonomia dos enfermeiros.

O fato da pesquisa ter sido desenvolvida em um único contexto pode ter se configurado como limitação. Daí a importância de outros estudos com maior abrangência para visualizar contextos e realidades diferentes.

\section{REFERÊNCIAS}

1. Chaves LD, Solai CA. Sistematização da assistência de enfermagem: considerações teóricas e aplicabilidade. $2^{\mathrm{a}}$ ed. São Paulo (SP): Martinari; 2013.

2. Sasso GTM, Barra DCC, Paese F, Almeida SRW, Rios GC, Marinho MM. Processo de enfermagem informatizado: metodologia para associação da avaliação clínica, diagnósticos, intervenções e resultados. Rev Esc Enferm USP [Internet]. 2013 [acesso em 2017 mar 15];47(1):242-9. Disponível em: http://www.scielo.br/pdf/reeusp/v47n1/a31v47n1.pdf.

3. Tannure MC, Lima APS, Oliveira CR, Lima SV, Chianca TCM. Processo de enfermagem: comparação do registro manual versus eletrônico. J Health Inform [Internet]. 2015 [acesso em 2017 mar 15];7(3):69-74. Disponível em: http://www.jhi-sbis.saude.ws/ojs-jhi/index.php/jhisbis/article/view/337.

4. Medeiros AL, Santos SR, Cabral RWL, Silva JPG, Nascimento, NM. Avaliando diagnósticos e intervenções de enfermagem no trabalho de parto e na gestação de risco. Rev 


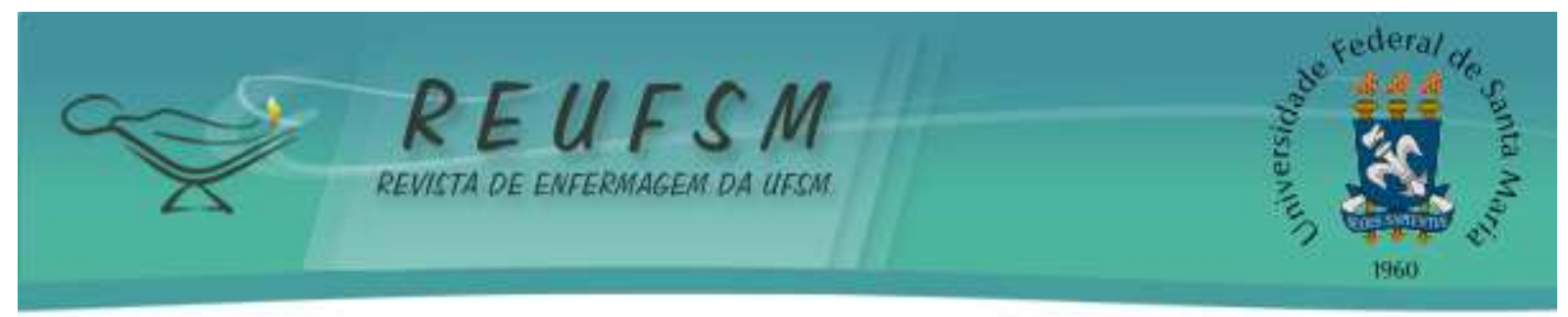

Gaúcha Enferm [Internet]. 2016 [acesso em 2017 mar 17];37(3):e55316. Disponível em: http://www.scielo.br/pdf/rgenf/v37n3/0102-6933-rgenf-1983-144720160355316.pdf.

5. Soares MIS, Terra FS, Oliveira LS, Resck ZMR, Esteves MSD, Moura CC. Processo de enfermagem e sua aplicação em Unidade de Terapia Intensiva: revisão integrativa. Rev Enferm UFPE [Internet]. 2013 [acesso em 2017 mar 18];7(N Esp):4183-91. Disponível em: https://periodicos.ufpe.br/revistas/revistaenfermagem/article/download/11647/13754.

6. Pereira MM, Germano RM, Câmara AG. Aspectos da assistência de enfermagem em Unidade de Terapia Intensiva. Rev Enferm UFPE [Internet]. 2014 [acesso em 2017 abr 18]; 8(3):545-54.

Disponível

em:

https://periodicos.ufpe.br/revistas/revistaenfermagem/article/download/9709/9782.

7. Santos DMA, Sousa FGM, Paiva MVS, Santos AT, Pinheiro JMS. A enfermagem baseada em evidências apoiando a construção do histórico de enfermagem: uma pesquisa bibliográfica. Cienc Cuid Saude [Internet]. 2016 [acesso em 2017 maio 30];15(3):561-9. Disponível em: http://periodicos.uem.br/ojs/index.php/CiencCuidSaude/article/view/26357.

8. Santos DMA, Sousa FGM, Paiva MVS, Santos AT. Development and implementation of a nursing patient history in pediatric intensive care. Acta Paul Enferm [Internet]. 2016 [acesso em 2017 maio 30];29(2):136-45. Disponível em: http://www.scielo.br/pdf/ape/v29n2/19820194-ape-29-02-0136.pdf.

9. Ceia MLM, Cesar JA. Avaliação do preenchimento dos registros de puericultura em unidades básicas de saúde em Pelotas, RS. Rev AMRIGS [Internet]. 2011[acesso em 2017 abr 14];55(3):244-9. Disponível em: http://repositorio.furg.br/handle/1/2509.

10. Brasil. Ministério da Saúde. Conselho Nacional de Saúde. Resolução CNS no 466, de 12 de dezembro de 2012. Aprova diretrizes e normas regulamentadoras de pesquisa envolvendo seres humanos. Brasília: Ministério da Saúde; 2012.

11. Arruda EHP, Ortiz TA, Pinheiro DO. Importância do autoconhecimento dos grupos sanguíneos (ABO e Rh) de alunos de Tangará da Serra-MT. J Health Inform [Internet]. 2013 [acesso em 2017 abr 18];15(3):199-202. Disponível em: http://pgsskroton.com.br/seer/index.php/JHealthSci/article/view/671.

12. Tavares TS, Castro AS, Figueiredo ARF, Reis DC. Avaliação da implantação da sistematização da assistência de enfermagem em uma unidade pediátrica. Rev Min Enferm [Internet]. 2013 [acesso em 2017 mar 19];17(2):278-86. Disponível em: http://www.reme.org. br/artigo/detalhes/650.

13. Morais CGX, Batista SEM, Castro GMO. Registros de enfermagem em prontuário e suas implicações na qualidade assistencial segundo os padrões de acreditação hospitalar: um novo olhar da auditoria. Rev ACRED. 2015;5(9):64-84.

14. Melo EM, Sales ICF, Almeida DV. Avaliação dos registros de enfermagem no balanço hídrico de pacientes em Unidade de Terapia Intensiva. Rev Enferm UFPI. 2014;3(4):35-41.

15. Saraiva EL, Sousa CS. Pacientes críticos na unidade de recuperação pós-anestésica: revisão integrativa. Rev SOBECC. 2015;20(2):104-12.

16. Padilha EF, Haddad MCF, Matsuda LM. Qualidade dos registros de enfermagem em terapia intensiva: avaliação por meio da auditoria retrospectiva. Cogitare Enferm [Internet]. 


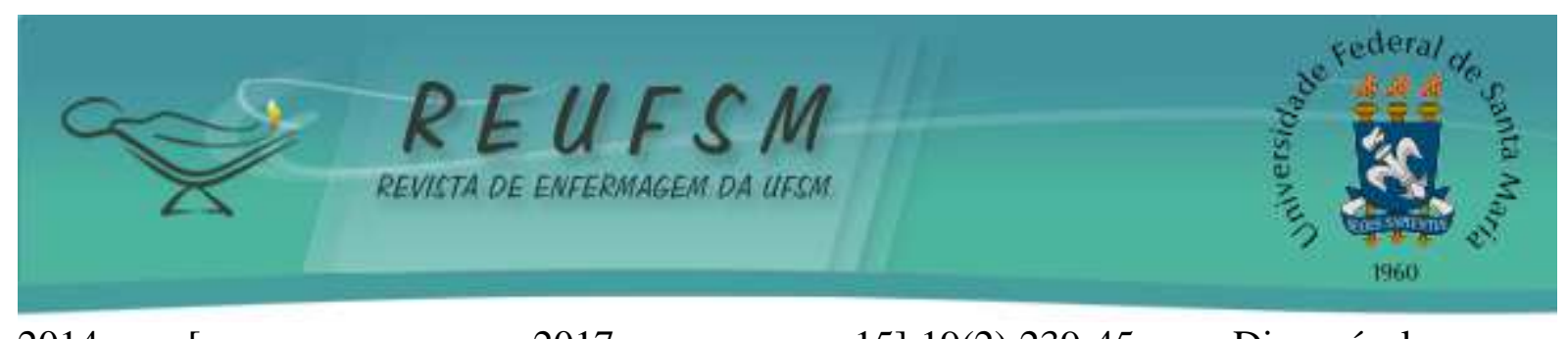

2014 [acesso em 2017 mar 15];19(2):239-45. Disponível em: http://pesquisa.bvs.br/brasil/resource/pt/lil-748006.

17. Maziero VG, Vannuchi MTO, Haddad MCL, Vituri DW, Tada CN. Qualidade dos registros dos controles de enfermagem em um hospital universitário. Rev Min Enferm [Internet]. 2013 [acesso em 2017 mar 12];17(1):165-70. Disponível em: http://www.reme.org.br/artigo/ detalhes/587.

18. Ascari RS, Ascari TM, Vieira MAC, Silva OM. Anotações de enfermagem e auditoria: uma revisão integrativa da literatura. Rev Saúde Pública Santa Cat [Internet]. 2015 [acesso em 2017 maio 30];8(2):111-21. Disponível em: http://esp.saude.sc.gov.br/sistemas/revista/index.php/ inicio/article/viewArticle/302.

19. Bittencourt GKGD, Crossetti MGO. Habilidades de pensamento crítico no processo diagnóstico em enfermagem. Rev Esc Enferm USP [Internet]. 2013 [acesso em 2018 mar 21]; 47(2):341-7. Disponível em: http://dx.doi.org/10.1590/S0080-62342013000200010.

Data de submissão: 12/08/2017

Data de aceite: $23 / 04 / 2018$

Autor correspondente: Jaynara Menezes Sousa Pinheiro.

E-mail: enf_jaynara@yahoo.com.br

Endereço: Avenida Bahia s/n Condomínio Gran Village Turu 2, Casa 41, Chácara Brasil Turu, São Luís - Maranhão

CEP: 65.066-659 